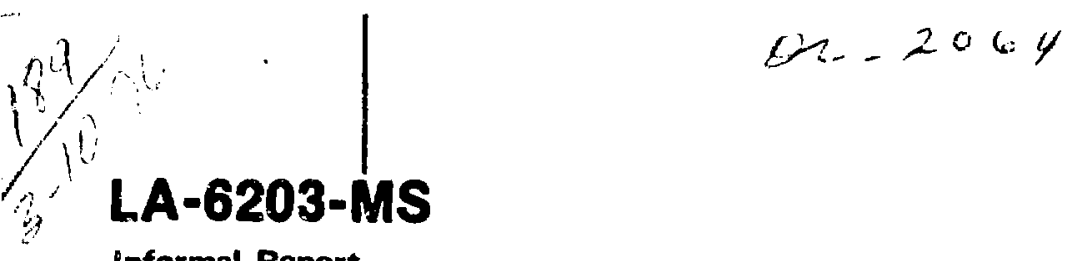

informal Report

UC-20

Reporting Date: January 1976

Issued: January 1976

\title{
An Ohmically Heated High-Density Z Pinch
}

by

Jay E. Hammel

- 011105

sciandiffe laboratory

of the University of California

los alAmos, New meXico 97545<smiles></smiles>

An Aflirmolive Action/Equal Opporlunity Employer 
In the interest of prompt distribution, this report was not edited by the Technical Information staff.

Printed in the United Statee of Americh. Avallable trom

National Technical information sevice

U.S. Deparment of Cpmmerce

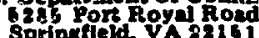

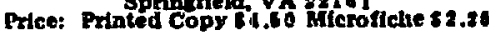

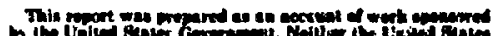

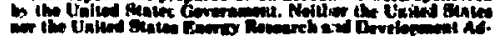

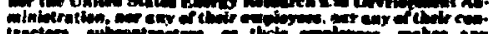

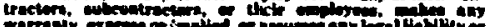

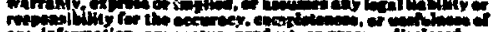

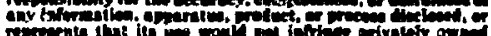

rithes. 
A⿱

by

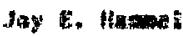

\section{corate}

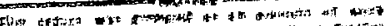

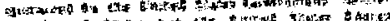

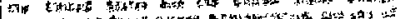
Fow

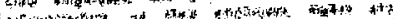

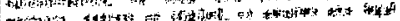

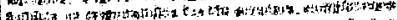
4 .

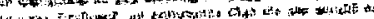
atritions

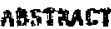

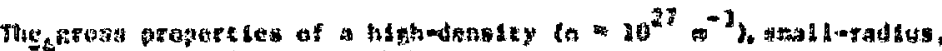

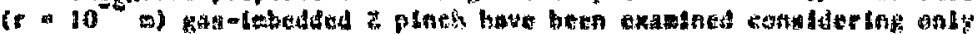

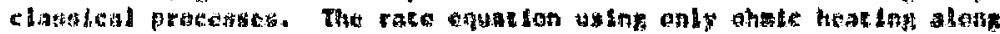

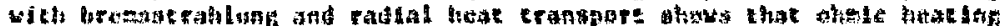

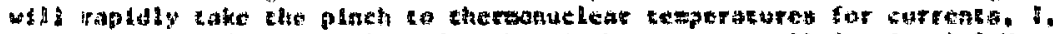

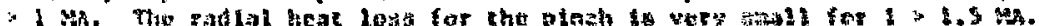

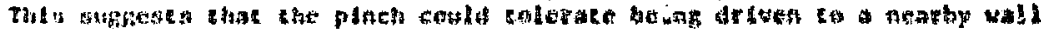
by at of 1 klak.

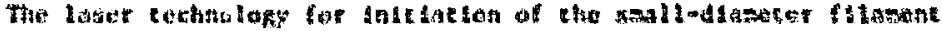

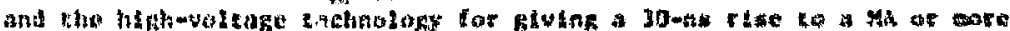

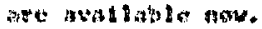

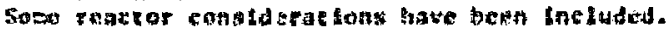

\section{1. thtroptetros}

The densisy rogthen of $10^{27} \mathrm{a}^{-1}$ has beas fitten

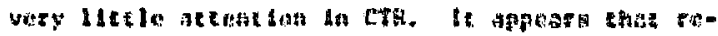

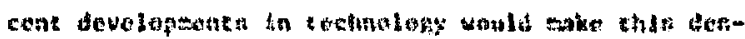

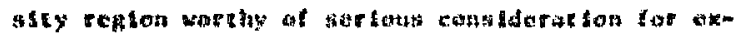

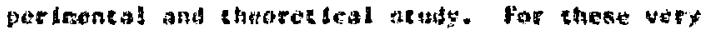

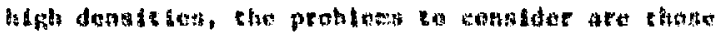

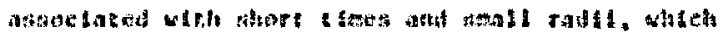

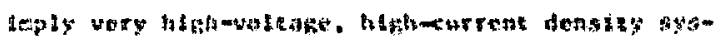

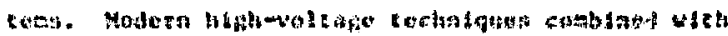

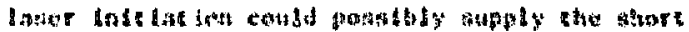

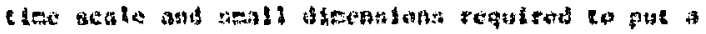

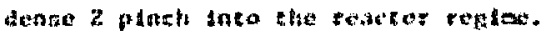

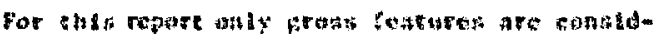

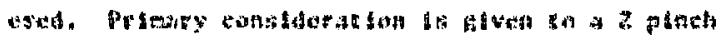

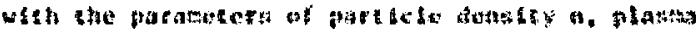

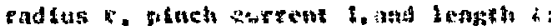

BAthe 1

$$
\begin{aligned}
& n_{1}+n_{0}=n=10^{27} a^{-3} \\
& 5=10^{-4} .
\end{aligned}
$$

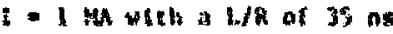

$$
\begin{aligned}
& n=10^{-1} \mathrm{~m}
\end{aligned}
$$

pinch eloe -100 ne

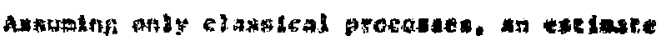

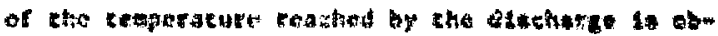

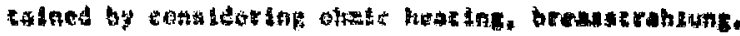
and radial that Iose.

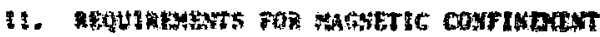

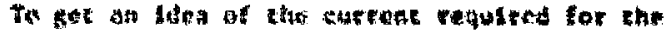

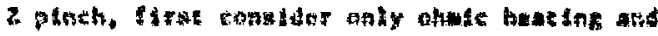
brematerandusa.

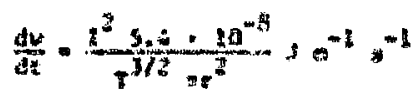

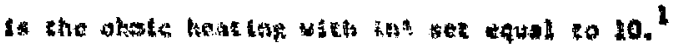

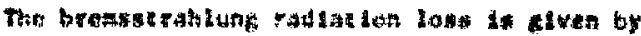

$$
\frac{d y}{d t}-6.0^{4} \cdot 10^{-17} n^{2} z^{-12} \cdot=\pi^{2}+n^{-1} \cdot 1
$$

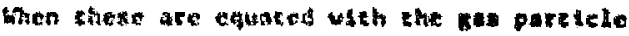

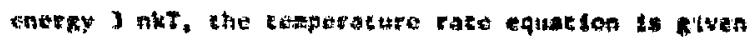
by

$$
\frac{d t}{d t}=\frac{3.3 \cdot 10^{8} t^{2}}{\left(n 5^{2}\right)^{2} n t^{3 / 2}}-3 \cdot 10^{-21} n t^{1 / 2}
$$




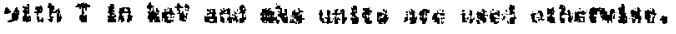

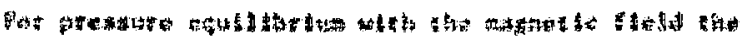

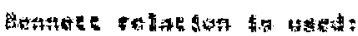

$27^{2}+1+10 \%$

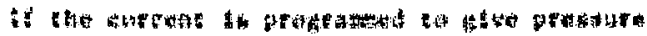

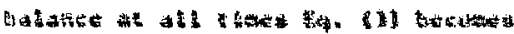

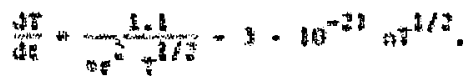

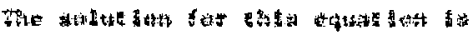

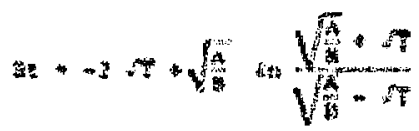

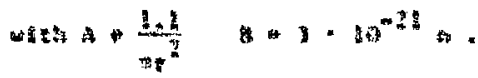

A phot of

Tha

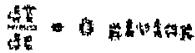

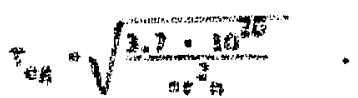

The Hot

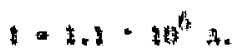

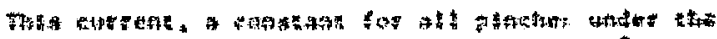

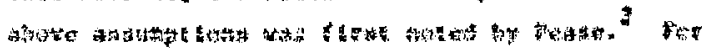

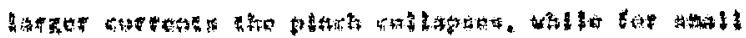

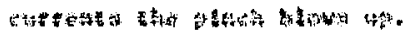

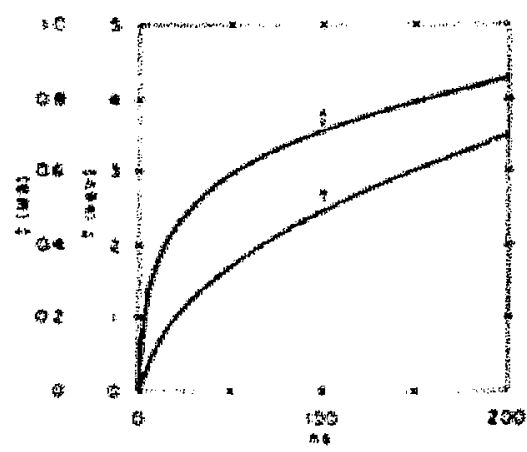

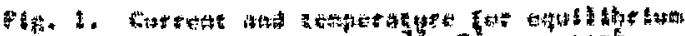

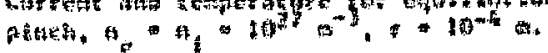

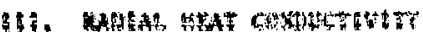

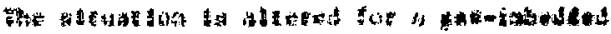

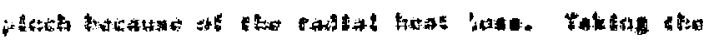

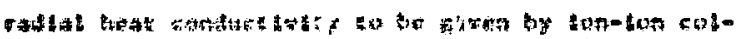

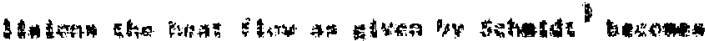

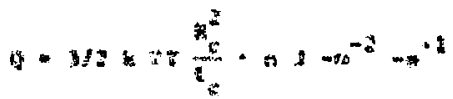

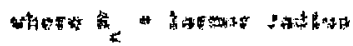

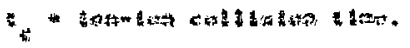

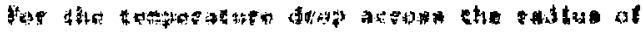

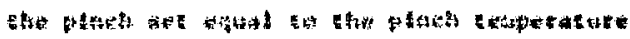

$$
\text { triction }
$$

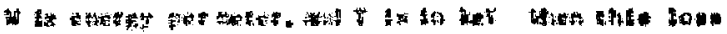
th a

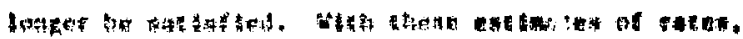

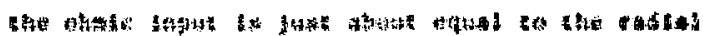

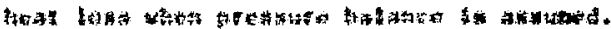

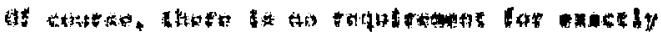

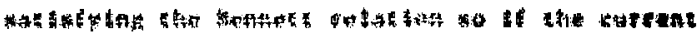

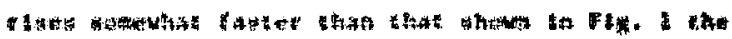

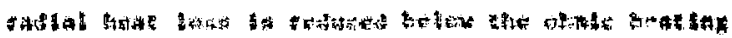

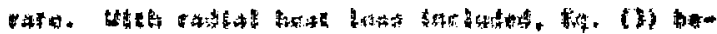

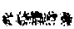

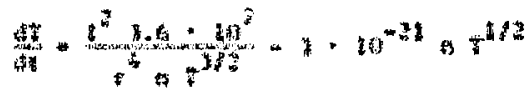

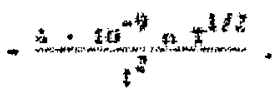

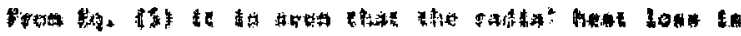

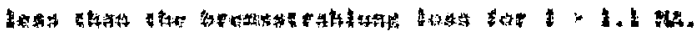

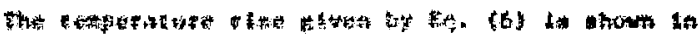
H.

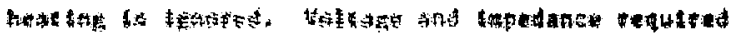

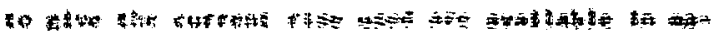

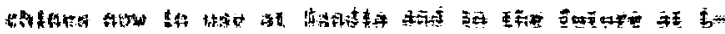

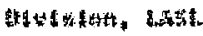

Tht 7-

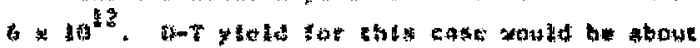

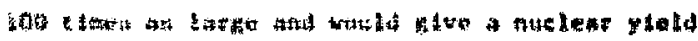

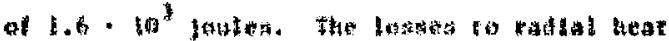




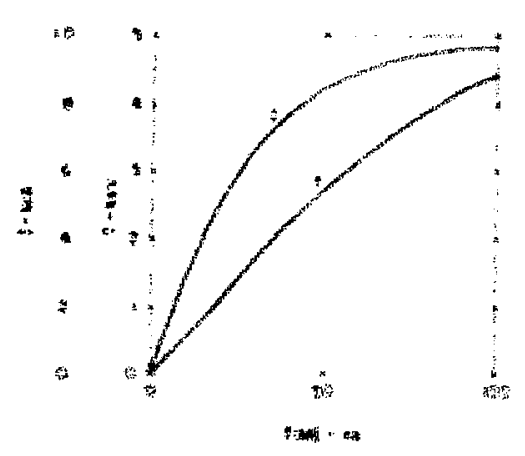

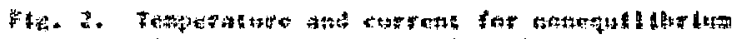

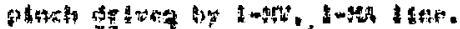
$13+10^{2} 2^{2}$

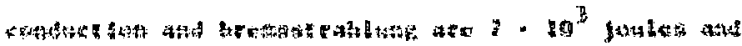

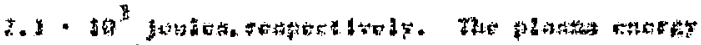

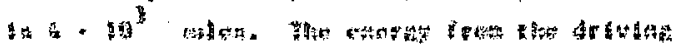

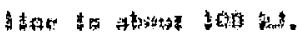

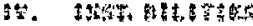

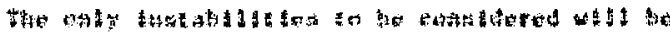

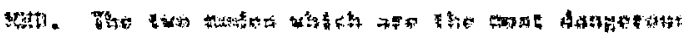

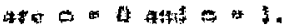

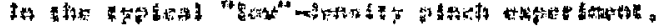

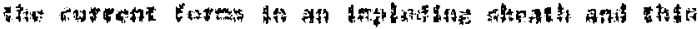
r.

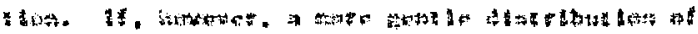

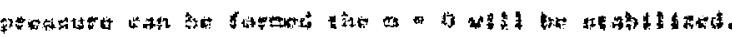

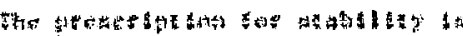

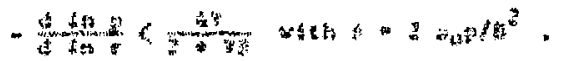

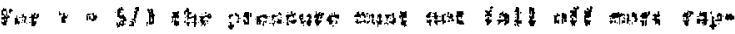

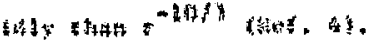

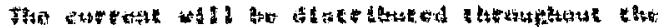

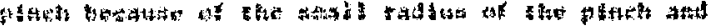

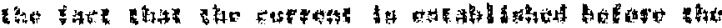

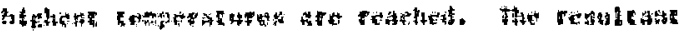

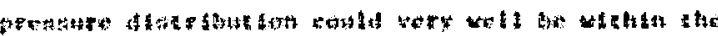
neable refath.

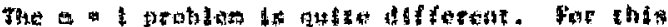

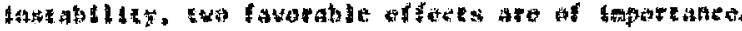

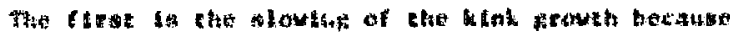

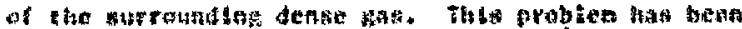

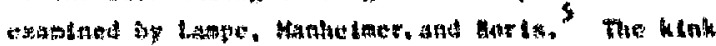

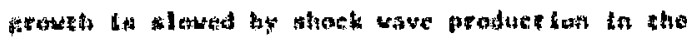

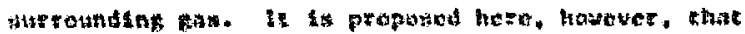

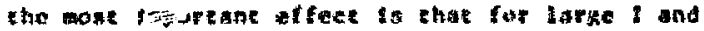

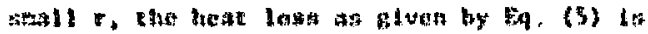

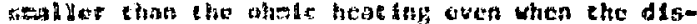

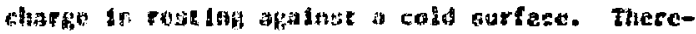

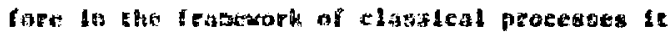

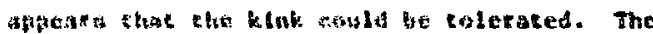

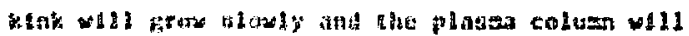

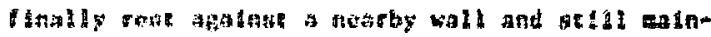

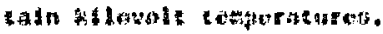

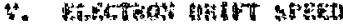

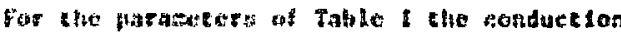

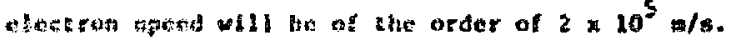

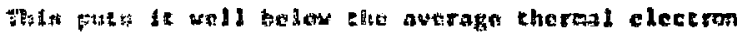

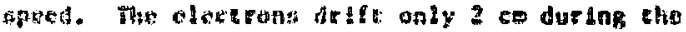

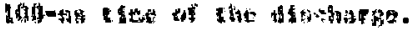

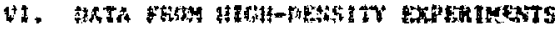

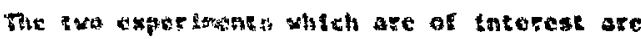

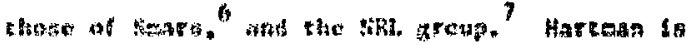
4.

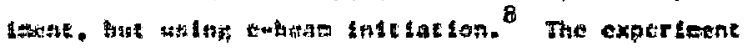

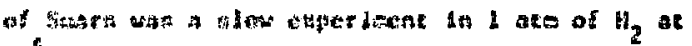

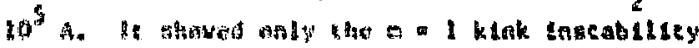

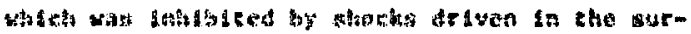

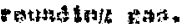

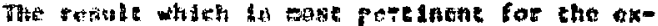

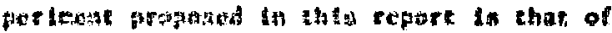

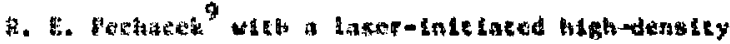

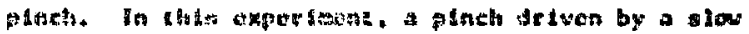

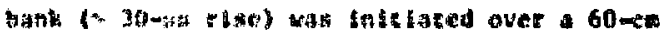

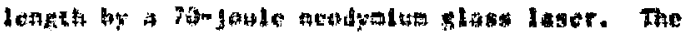

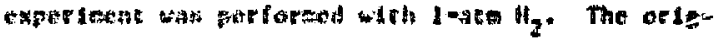

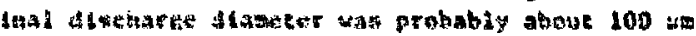

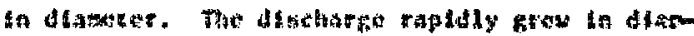

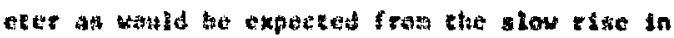

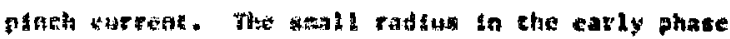

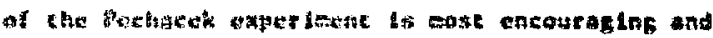

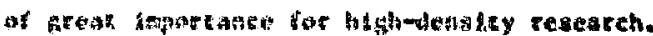

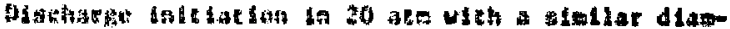

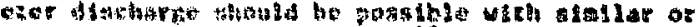

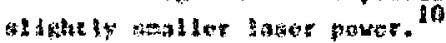

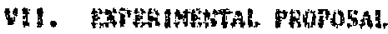

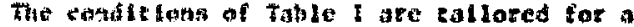

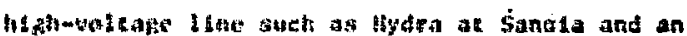

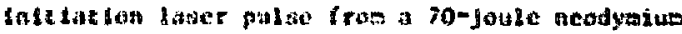

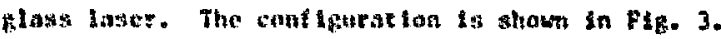
Tise size of che palyethyleos eyl under is ehat dorived from the $d$. C. Sartin notes for thasc experimencal esudtstonf, A.C., I M and $100 \mathrm{~ns}$. 


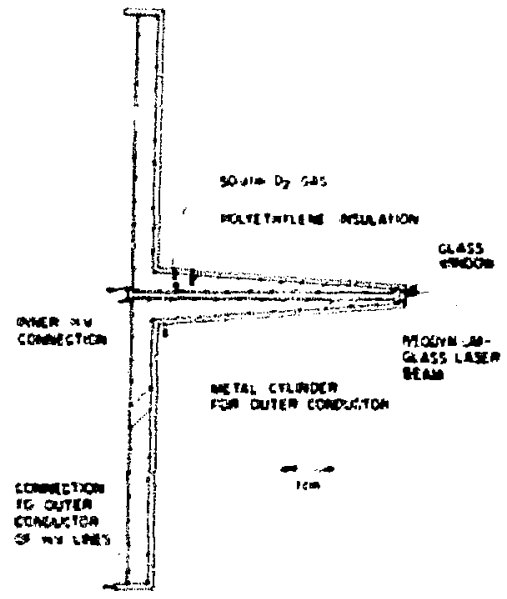

Fig. 3. Possible pinch configuration for 1-kN line.

The slepilcity of the expertment lat ezident; however, wany laportant facto could be deforalned frop chis modest effore. Of great importance are:

1. Intetation of the digcharge and resultant pinch dianezer

2. Stability of the pinch and particulariy the effect of the kink on the dilucharge

3. The heat loss from the pinch and the resuleant corona ${ }^{11}$ gurroundtag the discharge

4. The effeces of nearby vall on plneh conealnacni

5. Tie many surpriges at this denstey.

\section{VIIL. MEACTOA SCALTHE}

A very rough ides of the current required for breakweven can be found frow equating ohole hioting wlth breasstrohlung loss since the rate salculotions [En. (6)] shes that in small radli the tempersture will be appranched within the assumed pinch tate. The radlat heat loss is neglected above $2.5 \mathrm{MA}$. this glvee the relation betreen 1 ori n with a rem quired sesperature of $10 \mathrm{kev}$.

$$
n^{2} \cdot \frac{I^{2}}{r^{4}} 1.2 \cdot 10^{26} .
$$

The nuclear yfeld for D-T is

$$
\begin{aligned}
& Y=1 / 2 n^{2}(a v) \text {. (chergy/reaction) rol t } \\
& =1 / 2 n^{2}\left(11 \cdot 10^{-17} \cdot 10^{-6}\right) \\
& =19 \cdot 20^{6} \cdot 1.6 \cdot 10^{-19} \text { vo: } \cdot \\
& =\frac{7.2 \cdot 10^{-8}}{r^{2}} \cdot t 1^{2}
\end{aligned}
$$

The input energy goes primarily inco the inductance of the pinch. Thlo energy 18 given by

$$
w_{I n}-1 / 2 L_{a} R \cdot I^{2} \text {. }
$$

Th18 wakes a $\mathrm{Y} / \mathrm{H}_{\mathrm{In}}$ rat Io

$$
\begin{gathered}
\frac{Y}{W_{\text {In }}}=\frac{i .4 \cdot 10^{-7} t}{r^{2} L_{0}} . \\
\text { For } r=10^{-4} \pm . L_{0}=0.7 \mu H=\mathrm{m}^{-1} \\
\frac{Y}{W_{\text {In }}}=2.10^{7} \mathrm{t} .
\end{gathered}
$$

The yleld to input ratio being proportlonal to time 1s the result if the pinch is crowbarred at degign current. For energy in the fleld equal to yleld,

$\mathrm{c}=50 \mathrm{~ns}$.

Ueing the scallng as a guida, the following sample reactor parameters were cal sulated.

$$
\begin{aligned}
n & =2.5 \cdot 10^{27} \mathrm{~m}^{-3} \\
r & =10^{-4} \mathrm{~m} \\
\mathrm{r} & =5 \mathrm{kV} \\
I_{\max } & =5 \mathrm{kh}
\end{aligned}
$$

Length = $50 \mathrm{~cm}$

The resules of the actual calculation for the cemperature, yleld, etc., with accual clrcult cond1clons are

$$
\begin{aligned}
& \mathrm{T}-13.8 \mathrm{keV} \\
& \text { Nuclear yleld }=6.5 \mathrm{MJ} \\
& \text { Plasma enerby - } 17 \mathrm{~kJ} \\
& \text { Energy In field } * 2.5 \mathrm{~kJ} \\
& \text { Radial heat } 1095=1 \mathrm{~kJ} \\
& \text { Breasstrahlung loss }-6.5 \mathrm{~kJ}
\end{aligned}
$$

End loss by streaming in the 0 field to glower than a fleid-íte streaning and perhaps tew percent end 10,ss to to be expected in the 150me concalneent clece in the $0.5-\mathrm{m}$ plnch length.

This report cannot give reactor Jesign considerat sons other than the most elementary coments. certaln features can he tatilated:

1. The yleld co Input rat to 18 very favorable for this stallim radius, higi-density pinch.

2. The 5-MV, 5-Mh high-vol tage sourceg agsuked in the calculation do not require a great advance over chose now in existence.

3. Radiation damage problems are not severe becauge renewable parts are reasonable: in guch a small volume. 
4. Reactor feasibility experiments are not greatly different in size from reasonable first experiments.

IX. CONCLUSIONS

The great udvantage in CTR of an ohmically heated, higi-density pinch is evident. of course. many questions on the feasibllity of such a system cannot be answered with present data. The experiments needed to favestigate the high-density pinch are not expensive and do not require extraordinary advances in technology. The laser initiation phase could be done on a modes: scale with neodymium glass lasers now avallable at IASL. The first phase of a high-voltage discharge experiment could also be done with a slight modification to present e-beam power stpplies at Sandia or even at LASL.

It. Is felt that the main quest lons (can the small-dfameter pinch be established and can it survive the kiak by means of magnetic fleld insula(ion?) will be answered only by experiment.

\section{REFERENCES}

1. C. Longmire, Elementary Plasma Physics (Interscience Pubitshers), P. 176.

2. R. S. Pease, Proc. Phys. Soc., Vol. 40. January 1957).

3. G. Schmidt, Physics of High Temren ature Plasmas (Academtc Press, New York, 1966), p. 319

4. B. B. Kadonteev, in Revicus of Plasma Phystcs, Vol. 2, H. Leontovich, Ed. (Consulcants Bureau, New York, 1966).

5. W. Manheimer, M. Lampe, and J. Bor1s, NRL Mezorandum Report 2487 (August 1972).

6. E. A. Smärs, in Revinws of Plasma Physies, Vol. I, M. Leoneovich, Ed. (Consultants Buresu, Niew York, 1965), p. 205 .

7. R. Pechacek, Bull. As. Phys, Soc. 17, 593 (1972).

8. K. Struve, C. W. Hartman, R. Munger, and J. W. Sheaver, Bull. An. Phys. Soc, Soc. II, Vol. 20, B. 1296 (1975).

9. R. Pechacek, NRL, personal corrsuntention, 1975.

10. Day 1d Copeland, NRL, persional comsuntcation. 1975.

11. D. A. Tidman, "Structure of a Gas-Embedded 2 pirch Initiated Along a Laser-praduced Ionization Ellatent," Versar, Inc. (June 1971). (This report discusses the corona and many of the features of the pinch discussed here.) 